\title{
CONTRIBUIÇÕES DAS NEUROCIÊNCIAS PARA A FORMAÇÃO DE PROFESSORES
}

\author{
Crislaine Batista do Sacramento ${ }^{1}$ \\ Margeylson Ribeiro da Graça ${ }^{2}$
}

\begin{abstract}
Resumo
Este trabalho tem por objetivo descrever, com base em publicações nacionais, quais as contribuições que as neurociências podem proporcionar à formação de professores e sua prática pedagógica. Métodos: Para a realização deste estudo foi feita uma pesquisa qualitativa na qual foi realizado estudo bibliográfico onde serão coletadas publicações sobre o tema e em seguida analisar as contribuições teóricas de vários autores. Este estudo contribui para compreender quais subsídios as neurociências podem ofertar para a prática docentes, uma vez que se compreende é de suma importância que o docente conheça ou ao menos tenha noções do funcionamento do cérebro, para que então possa buscar estratégias e estímulos que facilitem a aprendizagem do aluno bem como contribua para a melhoria do processo educativo e organização da sala de aula, visto que ao saber como o cérebro funciona e quais recursos podem facilitar o trabalho a práxis pedagógica tornar-se-á mais significativa e eficaz no seu papel. Resultados: Os resultados levantados por esta pesquisa apontam que as neurociências têm contribuído de forma muito expressiva para o avanço do processo educativo e para a construção de uma aprendizagem significativa dentro da escola
\end{abstract}

Palavras-chave: Neurociências; Neuroeducação; Prática pedagógica.

\begin{abstract}
This paper aims to describe, based on national publications, what contributions neurosciences can provide to teacher training and its pedagogical practice. Methods: In order to carry out this study, a qualitative research was carried out in which a bibliographic study was carried out, where publications on the topic will be collected and then analyze the theoretical contributions of several authors. This study contributes to understand what subsidies neurosciences can offer to the teaching practice, once it is understood, it is of utmost importance that the teacher knows or at least has notions of the functioning of the brain, so that he can then seek strategies and stimuli that facilitate the student learning as well as contributing to the improvement of the educational process and organization of the classroom, since knowing how the brain works and what resources can facilitate the work, pedagogical praxis will become more meaningful and effective in its role. Results: The results obtained by this research show that neurosciences have contributed in a very expressive way to the advancement of the educational process and to the construction of meaningful learning within the school.
\end{abstract}

Keywords: Neuroscience; Neuroeducation; Pedagogical practice.

\footnotetext{
1 Mestra em Ciências da Educação pela FICS. Especialista em Educação Profissional Científica e Tecnológica pelo IFBA; Especialista em Neuroaprendizagem pela Unyleya. Especialista em Gestão Escolar e Coordenação Pedagógica pela Unyleya. Licenciada em Pedagogia pela UNIFACS. Coordenadora Pedagógica em São Francisco do Conde/Ba. Docente Escola Tercia Borges - Candeias/Ba. Email: crislaine.bs@hotmail.com.

2 Mestre em Ciências da Educação pela FICS, Especialista em Docência Superior, Licenciado em Matemática, Bahcarel em Sistemas de Informação, Coordenador da Licenciatura em Computação do Instituto Federal de Educação, Ciência e Tecnologia da Bahia e Professor de Programação do IFBA. Email: margeylsongraca@ifba.edu.br.
} 


\section{Introdução}

A formação dos professores cada vez mais tem sido um tema discutido entre pesquisadores da área, pois se compreende que existe uma necessidade de aprimoramento permanente, pois a sociedade atual passa por mudanças constantes e os docentes precisam acompanhar o avanço que se tem a todo instante, já que professores que buscam incessantemente formas de exercerem melhor a sua prática pedagógica, conseguem contribuir melhor para o desenvolvimento dos seus educandos.

Para compreender melhor o cérebro e sua forma de aprender os professores podem contar com os conhecimentos do campo das neurociências já que a aprendizagem é um processo cognitivo e envolve diversas funções e atividades cerebrais. O funcionamento do cérebro é complexo e requer estudo e aprimoramento constante já que sempre há novas descobertas a serem compartilhadas e estudadas.

O tema abordado permite refletir sobre as metodologias e teorias utilizadas na educação. Os saberes adquiridos a partir dessas reflexões permitem compreender que a relação entre aprender e ensinar exige atualmente um conhecimento sobre o cérebro e o seu funcionamento. O objetivo deste estudo é identificar as contribuições que as neurociências podem proporcionar à formação de professores e sua prática pedagógica.

A escolha deste tema, se deu pela necessidade de compreender como esses conhecimentos neurocientíficos podem contribuir com a prática docente dos professores. Buscou-se também compreender como os professores conseguiriam acesso a estes conhecimentos pois, devido às constantes modificações da sociedade os conhecimentos necessitam cada vez mais de revalidação e atualização periódica. Vale salientar que este artigo é resultante da dissertação de mestrado da autora, pesquisadora do tema.

E para melhorar o trabalho dos professores faz-se necessária uma construção coletiva de saberes, pois a maioria dos profissionais conhece pouco sobre o cérebro e como estimulá-lo e por isso é valido destacar que aos mesmos devem se oportunizados tempo e espaço para aquisição de novos conhecimentos numa 
prática reflexiva, que os levem a ter uma postura de mudança. E nesse cenário conhecer o funcionamento do cérebro e entender que há maneiras que favorecem o aprendizado é essencial para que a prática pedagógica contribua para uma aprendizagem significativa e duradoura.

A neurociência e todas as suas contribuições devem ser trazidas para o ambiente escolar, pois as funções metais são pertinentes à aprendizagem e o seu estudo e compreensão favorece o trabalho do professor em oferecer uma aprendizagem significativa aos discentes.

\section{Breve Conhecimento Sobre a Neurociência}

Compreender a neurociência é o primeiro passo que seja possível aplicar os seus conhecimentos e descobertas em qualquer área existente e para tal, faz-se necessário primordialmente entender o conceito esse termo a fim de dar maior significado ao aprendizado, pois a neurociência precisa ser compreendida como um conjunto de ciências que investigam o sistema nervoso com grande interesse em compreender a atividade cerebral, pois:

\footnotetext{
Neurociência é o estudo do sistema nervoso, das suas composições moleculares e bioquímicas, e as diferentes manifestações deste sistema e do tecido, através das nossas atividades intelectuais, tais como a linguagem, o reconhecimento das formas, a resolução de problemas e a planificação das ações (NASCIMENTO, 2011, p. 24).
}

Essa afirmação traz à luz de que a neurociência envolve as mais diversas áreas do conhecimento e com isso percebe-se a multidisciplinaridade que envolve as neurociências, já que para que toda a ação humana aconteça é necessária uma ação cerebral. A neurociência muito tem contribuído para a compreensão e esclarecimento acerca do que acontece dentro do cérebro humano e desde a década de 90, também conhecida como Década do Cérebro, muito tem se buscado para compreender o cérebro e o seu funcionamento, desde exames computadorizados a diagnósticos por imagem, tudo para ver e compreender o cérebro em funcionamento.

A neurociência, enquanto campo de estudo que envolve diversas áreas acerca do estudo do sistema nervoso, do funcionamento e estrutura anatômica e fisiológica do cérebro, traz à luz para conhecimentos dos professores, explicações 
sobre o comportamento e o processo de aprendizagem e cognição humana, além dos mecanismos de regulação orgânica. Este campo de estudo fundamenta uma recente teoria da aprendizagem baseada nas novas descobertas e abordagens a respeito do cérebro e seu funcionamento que está transformando a concepção de aprendizagem, avaliação e currículo.

Essa gama de conhecimentos disponibilizados pelas neurociências auxilia 0 docente a compreender o que ocorre no cérebro quando este entra em contato como novos saberes e como o processo de aquisição do conhecimento acontece se tornando aprendizado significativo e duradouro.

\section{Contribuições das Neurociências Para a Formação e Papel do Professor}

O cérebro pode ser treinado, e isso requer o estímulo do professor e decisão do estudante em contribuir com a atenção para o processo construção de novos saberes. Desta maneira, por atuar diretamente com os educandos cabe muito ao professor interligar o conhecimento e as mudanças sociais com o aluno, promover a construção da subjetividade, estimular a construção de novos conhecimentos através do confronto de ideias e principalmente excitar e sustentar ações inclusivas realizadas com responsabilidade e respeito.

E para isso, a princípio é necessário que o docente tenha sua formação de acordo com o que define a LDBEN 9.394/96, a qual em seu artigo 62 define que "a formação de docentes para atuar na educação básica far-se-á em nível superior, em curso de licenciatura de graduação plena em universidade e instituição de educação". Pois é fundamental que para atuar como docente o profissional conheça as bases do processo educativo. Além da formação básica, o professor precisa buscar constante aprimoramento e aprendizagem dentre esses conhecimentos está a neurociência que vem auxiliar o docente na sua prática, pois:

\footnotetext{
Para compreender como a neurociência contribui com o processo de ensino é preciso conhecer a anatomia da aprendizagem e como as áreas do sistema nervoso são estimuladas e as informações são processadas. Por isso a importância de se conhecer as funções desenvolvidas pela região cerebral denominada córtex (GROSSI, LOPES E COUTO 2014, P.30).
}

As contribuições que as neurociências podem acrescentar a prática do docente o auxiliarão a contribuir para que o estudante se desenvolva da melhor 
forma possível e no tempo do educando. Pois, de conforme esclarece Soares (2003) "se o ensinante toma conhecimento deste funcionamento cerebral, pode ressignificar sua prática docente adotando uma didática que caminhe na forma sensório-motora ao funcionamento operatório formal." Pois a neurociência de acordo com Portes (2015) a neurociência auxilia o ensinante a tornar sua práxis mais significativa e eficiente, e nesse sentido:

É por demais relevante saber que nesse processo de produção do conhecimento significativo a própria estrutura cognitiva do indivíduo também se modifica ampliando-se, diversificando-se e intensificando seu potencial tornando-se assim cada vez mais capaz de processar novas informações, ideias e dados e ancorar os resultados desse processamento num continuum aparentemente ilimitado. (SILVA E BEZERRA, 2011, p.04).

O professor deve mediar as trocas dos estudantes com o meio, sem tentar padronizá-los e deve também incentivar atitudes de solidariedade e cooperação entre todos para que enfim possam ser construídas uma escola, uma educação e uma sociedade que sirva para todos. Pois de acordo com Silveira (2004, n.p.) Quando compreendem que aprendizagem envolve cérebro, corpo e sentimentos adotam uma ação mais competente levando em conta a influência das emoções para o desenvolvimento na construção do conhecimento, e:

Para entender o que ocorre com o cérebro quando uma pessoa aprende, faz-se necessário realizar releituras dos teóricos da educação, associandoos aos estudos da neurociência; compreender a biologia do cérebro nas dimensões cognitivas, emocionais, afetivas e motoras; reconhecer que o processo de aprender está relacionado com as bases químicas e físicas na função neural do ser humano; e, como cada ser humano é único, cada cérebro é único e aprende de forma diferente. Portanto, é preciso ensinar de formas diferenciadas (GROSSI, LOPES E COUTO 2014, P. 28-29).

Mas esse aprendizado por parte do professor não ocorre somente em cursos de capacitação ou especializações, mas também na troca de experiências, vivências diárias e experimentos, cabendo ao professor buscar sempre compreender um pouco mais sobre a complexidade do cérebro, pois,

As neurociências não propõem uma nova pedagogia e nem prometem solução para as dificuldades da aprendizagem, mas ajudam a fundamentar a prática pedagógica que já se realiza com sucesso e orientam ideias para intervenções, demonstrando que estratégias de ensino que respeitam a forma como o cérebro funciona tendem a ser mais eficientes (COSENZA e GUERRA, 2011, p.139). 
Precisa ser ofertado aos docentes informações acerca das descobertas neurocientíficas, para que estes possam relacionar conhecimentos da neurociência com a educação, e assim elaborar currículos fundamentados no desenvolvimento cerebral, e assim também aproveitem dos resultados dessa pesquisa para incrementar sua práxis pedagógica e facilitar a aprendizagem dos estudantes, pois as neurociências permitem um novo olhar e adaptações para que através dos mais variados estímulos a aprendizagem seja estimulada, favorecendo assim a consolidação da memória de longa duração, pois:

\begin{abstract}
Ao conhecer o funcionamento do sistema nervoso, os profissionais da educação podem desenvolver melhor seu trabalho, fundamentar e melhorar sua prática diária, com reflexos no desempenho e na evolução dos alunos. Podem intervir de maneira mais efetiva nos processos de ensinar e aprender, sabendo que esse conhecimento precisa ser criticamente avaliado antes de ser aplicado de forma eficiente no cotidiano escolar. Os conhecimentos agregados pelas neurociências podem contribuir para um avanço na educação, em busca de melhor qualidade e resultados mais eficientes para a qualidade de vida do indivíduo e da sociedade (COSENZA e GUERRA, 2011, p. 145).
\end{abstract}

$\mathrm{Na}$ atualidade, o papel do docente deixa de ser de transmissor de conhecimento para transformar-se em mediador buscando auxiliar o estudante na construção dos seus conhecimentos, e para isso o professor precisa estar atento às necessidades desse novo público, mas essa modificação causa dúvidas e incertezas para o educador que procura compreender o significado de seu papel e sua função frente às transformações atuais, pois,

Os avanços das neurociências possibilitam uma abordagem mais científica
do processo ensino-aprendizagem, fundamentada na compreensão dos
processos cognitivos envolvidos. Devemos ser cautelosos, ainda que
otimistas em relação às contribuições recíprocas entre neurociências e
educação[...] Descobertas em neurociências não autorizam sua aplicação
direta e imediata no contexto escolar, pois é preciso lembrar que o
conhecimento neurocientífico contribui com apenas parte do contexto em
que ocorre a aprendizagem. Embora ele seja muito importante, é mais um
fator em uma conjuntura cultural bem mais ampla (lbid, p. 136).

Desta forma, percebe-se que as neurociências são fortes aliadas à melhoria da práxis docente, pois auxilia o profissional na busca de estratégias que favoreçam a aprendizagem do estudante contextualizando as descobertas deste campo com a realidade da sua turma. E esses conhecimentos podem ajudar o professor na compreensão e alternativas para lidar com dificuldades de aprendizagem, falta de atenção, pouca compreensão, dentre outros problemas encontrados no cotidiano de uma sala de aula. 
Os saberes advindos das neurociências possibilitam uma ampla e profunda concepção do funcionamento da mente humana permitindo que o professor busque didáticas e metodologias diferentes a fim de estimular a neuroplasticidade, favorecer o processo de ensino aprendizagem, atendendo às singularidades de um grande número de educandos. As mudanças possibilitadas pelas neurociências são diversas, mas será necessário tempo para que os docentes se apropriem destes conhecimentos e possam incluí-los em sua prática.

\section{Considerações}

Tendo como pressuposto que a educação ocupa papel fundamental na transformação da sociedade e que cabe a ela possibilitar a construção do conhecimento tem-se o professor como principal agente de transformação, assim volta-se para ele novas exigências e mais do que nunca o educador precisa estar habilitado não só em relação aos conhecimentos do mundo mais em relação aos conhecimentos curriculares e pedagógicos.

Sendo assim, a neuroeducação traz conhecimentos essenciais para a promoção de uma aprendizagem significativa e esta por sua vez possibilita que a escola desempenhe seu papel social que é de formar cidadãos capazes de transformar a sociedade tornando-a mais democrática.

Mas para estar desempenhando seu papel cada vez de maneira mais eficaz, é de primordial importância que o docente conheça ou ao menos tenha noções do funcionamento do cérebro, para que então possa buscar estratégias e estímulos que facilitem a aprendizagem do aluno bem como contribuía para a melhoria do processo educativo e organização da sala de aula, visto que ao saber como o cérebro funciona e quais recursos podem facilitar o trabalho a práxis pedagógica tornar-se-á mais significativa e eficaz no seu papel.

Neste cenário, a neuroeducação tem contribuído de forma muito expressiva para o avanço do processo educativo e uma aprendizagem significativa dentro da escola. Pois, o desenvolvimento do ser humano é um processo dinâmico e contínuo, não acaba com o passar dos anos, o que acontece é que em cada idade os objetivos são diferentes. Esse desenvolvimento ocorre interligado com os fatores pessoais e sociais. A neuroeducação tem trazido para dentro da realidade escolar 
saberes acerca do funcionamento e estrutura do cérebro para que a escola possa melhor cumprir sua função social de auxiliar na formação de cidadãos.

A formação continuada com base nas descobertas das neurociências, não pode ser vista como um conjunto de métodos e/ou conteúdos a serem seguidos como uma solução a todos os problemas da educação, porém deve servir como uma interação dos pressupostos teóricos, a prática pedagógica e a realidade da escola, onde os professores conscientes de que ambos devem caminhar juntos, pois a teoria o ajuda a refletir sobre sua prática dando-Ihe sentido e a prática demonstrando a necessidade da teoria a fim de fundamentar-se.

\section{Referências}

BRASIL. MEC. Lei de Diretrizes e Bases da Educação Nacional. No. 9394, de 20 de dezembro de 1996.

COSENZA, R. M; GUERRA, Leonor B. Neurociência e educação: como o cérebro aprende. Porto Alegre: Artmed, 2011.

GROSSI, M. G. R.; LOPES, A. M. COUTO. P. A., A Neurociência Na Formação De Professores: Um Estudo Da Realidade Brasileira. Revista da FAEEBA Educação e Contemporaneidade, Salvador, 2014.

MONTEIRO. J.J. Formação docente, caminhos possíveis à luz da neurociência., 2011, 70 f. Trabalho de Conclusão de Curso (Especialização) Rio de janeiro. Disponível em: http://www.avm.edu.br/docpdf/monografias publicadas/c205683.pdf. Acesso em: 26 dez. 2016

NASCIMENTO, M.J.S. O papel da neurociência no processo de aprendizagem. 2011. http://www.avm.edu.br/docpdf/monografias publicadas/C206339.pdf. Acesso em: 16 mar. 2017

NOGARO, A. Neurociência, formação de professores e práticas pedagógicas. 2012. Disponível em: http://www.webartigos.com/artigos/neurociencia-formacao-deprofessores-e-praticas-pedagogicas/90118/ . Acesso em: 26 dez. 2016

OLIVEIRA, G.G. Neurociência e os processos educativos: Um saber necessário na formação de professores. 2011.

PORTELA, G. L. Abordagens teórico-metodológicas. Projeto de Pesquisa no ensino de Letras para o Curso de Formação de Professores da UEFS. Feira de Santana-BA. 2004

PORTES, D. S. A importância das Neurociências na formação do professor de 
inglês. 2015. Disponível em: http://pepsic.bvsalud.org/pdf/psicoped/v32n98/07.pdf. Acesso em: 26 dez. 2016

SILVA, E. L.; MENEZES, E. M. Metodologia da pesquisa e elaboração de dissertação. 4. ed. Florianópolis: UFSC, 2005.

SILVA, F. MORINO, C.R.I A importância das neurociências na formação de professores. Momento - Diálogos em Educação, Rio Grande, v. 21 n. 1, p.29-50, 2012. Disponível em: https://www.seer.furg.br/momento/article/view/2478/2195. Acesso em: 26 dez. 2016

SILVA, M.M.; BEZERRA, E.L. Contribuições das neurociências ao processo de ensino-aprendizagem. Artigo apresentado ao $\mathrm{V}$ Colóquio Internacional de educação e Contemporaneidade, São Cristóvão, Sergipe. 2011. Disponível em: http://educonse.com.br/2011/cdroom/eixo\%2014/PDF/Microsoft\%20Word\%20-

\%20CONTRIBUIcoES\%20DAS\%20NEUROCleNCIAS.pdf. Acesso em: 26 dez. 2016

SILVEIRA, M.M.S. O Funcionamento do Cérebro no Processo de Aprendizagem. 2004. Disponível em: http://www.profala.com/arteducesp52.htm. Acesso em: 24 fev. 2017

SOARES, D.C. $\mathbf{O}$ cérebro $\mathbf{x}$ aprendizagem. 2005. Disponível em: http://www.profala.com/arteducesp67.htm. Acesso em: 24 fev. 2017

SOARES, D.C. Os Vínculos como passaporte da Aprendizagem: Um encontro D' EUS. Rio de Janeiro. Caravansarai, 2003. 